\title{
LUT
}

University

\section{Sensitivity analysis of a PLC-based DSSS anti-islanding system in power distribution grids}

Poluektov Anton, Pinomaa Antti, Romanenko Aleksei, Ahola Jero, Kosonen Antti

This is a Final draft

version of a publication

published by Elsevier

in International Journal of Electrical Power \& Energy Systems

DOI: $10.1016 / j . j$ jepes.2019.06.022

Copyright of the original publication: ( 2019 Elsevier

Please cite the publication as follows:

Poluektov, A., Pinomaa, A., Romanenko, A., Ahola, J., Kosonen, A. (2019). Sensitivity analysis of a PLC-based DSSS anti-islanding system in power distribution grids. International Journal of Electrical Power \& Energy Systems, Vol. 113. pp. 739-747. DOI: 10.1016/j.ijepes.2019.06.022

This is a parallel published version of an original publication. This version can differ from the original published article. 


\title{
Sensitivity Analysis of a PLC-Based DSSS Anti-Islanding System in Power Distribution Grids
}

\author{
Anton Poluektov, Antti Pinomaa, Aleksei Romanenko, Jero Ahola, and Antti Kosonen
}

\begin{abstract}
As smart grid applications, distributed generation, and microgrid technologies have become more widespread, new safety-related issues have arisen. Unintentional islanding is an example of a grid fault that may result in damage to electrical equipment and severe personal injuries. In this paper, an antiislanding system employing power line communication (PLC) and direct-sequence spread spectrum (DSSS) modulation implemented with software-defined radios for continuous signaling is tested in a laboratory test setup. A concept of a fault detection algorithm is evaluated. A DSSS sensitivity analysis is carried out to investigate system's performance against variation of a signalto-noise ratio, and define optimal DSSS settings. The results of the analysis are interpreted, and conclusions are drawn. A transformer bypassing scheme allowing to increase the fault detection speed and throughput is introduced and tested, and the conditions of applicability are described. As a result of the study, recommendations for the system development are presented.
\end{abstract}

Keywords-Direct-sequence spread spectrum, distributed power generation, islanding, loss of mains, power line communication, sensitivity analysis, software-defined radio.

\section{NomenClature}

$B \quad$ Signalling bandwidth

C Channel capacity

$\alpha \quad$ Signal attenuation coefficient

$\mu \mathrm{G} \quad$ Micro grid

BER Bit error rate

CDMA Code division multiple access

DG Distributed generator

DSSS Direct-sequence spread spectrum

ID Islanding detection

LoM Loss of mains

LV Low voltage

MV Medium voltage

NB Narrow band

NDZ Non-detection zone

OFDM Orthogonal frequency division multiplexing

PC Personal computer

PLC Power line communication

Rx Signal receiver

SC Spreading code length

SDR Software-defined radio

SG Smart grid

SK Synchronization key length

SNR Signal-to-noise ratio

Authors are with LUT School of Energy Systems, LappeenrantaLahti University of Technology LUT, Lappeenranta, Finland e-mail: (see https://www.lut.fi/web/en/school-of-energy-systems/contact).
THD Total harmonic distortion

Tx Signal transmitter

Tx/Rx Transceiver, intermediate signal repeater

USRP Universal Software Radio Peripheral, a brand name

VI Virtual instrument

\section{INTRODUCTION}

\section{A. Islanding condition and associated risks}

Recently, distributed generation (DG) together with micro and smart grid $(\mu \mathrm{G} / \mathrm{SG})$ technologies has attracted considerable attention and become widespread. Besides advanced features, i.e., grid monitoring and control, data transmission, improved energy efficiency, and system robustness, new safety-related issues have arisen. An unintentional islanding condition as a particular fault case is among the concerns to be addressed.

Islanding describes a condition where a part of the grid becomes disconnected from the main power utility, being energized by a distributed generation unit (DG). The condition may be unintentional, resulting for instance from environmental disturbances and a loss-of-mains (LoM) fault, and/or intentional when an island starts operating autonomously as a $\mu \mathrm{G}$. Unintentional islanding may cause harm to the grid personnel unaware of the island location or to the electrical equipment as a result of possible overvoltages and overcurrents [1]. Moreover, the grid power balance may be disturbed.

Islanding condition should be recognized in a shortest period of time in order to prevent an aftermath and switch the grid automatically to a controlled island mode, or disconnect DGs. An intentional island mode is applied to maintain grid operation under a fault condition and also requires grid state monitoring [2]-[6]. Introduction of DGs to electrical grids is regulated by standards institutions (e.g. IEEE and IEC) and by local regulating authorities [7]. Islanding detection (ID) is a mandatory functionality for a DG according to the IEEE standards 929-2000 and 1547-2003, which state that the unit should disconnect from the grid within $2 \mathrm{~s}$ in the case of islanding [8], [9].

\section{B. Islanding detection methods}

ID methods can be divided into three main categories; passive, active, and communication-based ones [1], [7], [10]. Hybrid solutions combine several ID methods, e.g. passive and active [11]-[13], passive and communication-based [5], [14]. The solutions vary by complexity, cost, fault detection accuracy, scalability, applicability and speed. Fault detection accuracy is commonly characterized by a non-detection zone (NDZ), which represents a range of conditions when an islanding condition is not detected by the safety system. 
Passive solutions are based on monitoring of sudden deviations in the characteristics of the supplied power, and the protection algorithm is triggered when a permitted fluctuation threshold is exceeded. Passive systems typically consider voltage, frequency, phase angle, particular harmonics, or total harmonic distortion (THD) [7], [15]-[20]. It is important to notice that when the power consumption of the local load nearly matches the output power of the DG, a passive solution may be ineffective [15], [18], [21]. In this case, the customers are mainly energized by the DG, and fluctuations of the monitored parameters of the supplied power are not sufficient to be recognized by the passive system; thus, the islanding condition is not detected [15], [21]. Passive solutions also have a range of conditions, under which an ID system detects a fault in a normal grid state. For relay-based systems, NDZ is affected, for instance, by dynamic voltage and frequency variations, a type of the DG (a/synchronous), or a type of DG controllers (constant current or power) [18], [22]. Passive systems have relatively large NDZ comparing to active and communicationbased solutions. For this reason hybrid passive solutions applying advanced techniques, and featuring machine-learning [1], neural networks [23], and pattern recognition [24] have been developed lately to achieve a higher ID efficiency.

Active systems apply artificial disturbance injection and grid feedback monitoring [1], [15], [25], [26]. Active systems have a much smaller NDZ, comparing to passive methods, but at the same time have certain drawbacks. First of all, injected disturbances decrease the power quality, and therefore are designed to be as small as possible. Furthermore, mutual interference in the case of a high number of DGs may decrease the fault detection accuracy [7], [15]. Islanding misdetection is possible when synchronous generators are operated with power electronic-based sources. Finally, active ID methods are typically developed for inverter-based DGs and are not applicable in case of synchronous generators [12].

Communication-based ID solutions do not depend on the type of a DG unit and therefore have a wider application range than active and passive ID systems. In contrast to active and passive schemes, they do not decrease the power quality and have a smaller NDZ [1]. Communication-based systems employ data transmission between protected devices and can be based on supervisory control and data acquisition (SCADA), power line communication (PLC), or communication with a utility recloser unit [5], [7], [14], [15]. Main challenges of this type of ID schemes is their relatively high cost, complexity, and operation speed. PLC-based solutions stand out, as the communication channel exists in the power lines, and thus no separate communication medium is needed. Application of a noise and attenuation robust signaling method, e.g. directsequence spread spectrum (DSSS), capable for long-distance signaling also minimizes expenditures on intermediate signaling units comparing to other PLC-based schemes.

\section{Contributions}

According to [15], PLC-based ID methods can detect a fault within $200 \mathrm{~ms}$. A PLC-based solution applying commercial hardware was presented in [27], [28]. According to the authors of these papers, the system provided signal reception in the range of approximately 4 miles $(6.4 \mathrm{~km})$. Data transmission was not considered to be an essential system functionality. The concept proposed in [29] has an ID time of approximately $20 \mathrm{~ms}$; however, this result was achieved by computer simulation and the concept was not evaluated neither during laboratory nor field tests. A hybrid solution combining voltage and frequency measurements with PLC signaling was proposed in [30]: the concept was tested in a medium-/low-voltage (MV/LV) grid. In the best case, an islanding was detected within $55 \mathrm{~ms}$, yet the maximum achieved signaling distance was only $1.2 \mathrm{~km}$. The signaling frequency band, error rate, transmitted data parameters, and signal processing algorithms were not covered, and wide-band modulation techniques were not discussed.

This paper introduces a state-of-the-art anti-islanding system, which applies continuous signaling on narrow-band (NB) PLC devices, and a DSSS modulation. The system is implemented and evaluated by using software-defined radios (SDRs). The work fills a niche and provides the following contributions to the field of PLC-based anti-islanding:

1) A novel ID algorithm, allowing also multiuser communication, is presented and evaluated in the laboratory. Thus, system's applicability for islanding detection is demonstrated in adequate conditions. ID speed fulfills IEEE standards 929-2000 and 1547-2003. Estimated signaling distance, calculated based on the channel attenuation coefficient [31], exceeds $10 \mathrm{~km}$. In both ID speed and signaling distance the proposed system excels competing solutions.

2) A sensitivity analysis of DSSS modulation settings is performed in the laboratory. The analysis covers a wider range of DSSS settings than the case presented in [32]. The target is to study the performance of the DSSS, i.e., sensitivity and selectivity against variation of the signalto-noise ratio (SNR) in normal and fault conditions. The DSSS spreading code length and the synchronization key length, which provide the highest fault detection speed and communication throughput under various SNRs, are determined.

3) The processing latency (affects the fault detection speed) of the system is investigated during communication tests. Affecting factors are described.

4) Extending the study of [32], a bypassing scheme is presented for the MV/LV transformer, and the operation results and the operation limits are evaluated by laboratory tests. The scheme is designed for the scenario where a signal cannot propagate through the MV/LV transformer because of a low SNR on the MV side, and increases the fault detection speed and throughput.

The proposed system is described in Section II. The fault detection system, the DSSS sensitivity analysis, and the bypassing scheme tests carried out in the laboratory setup are presented in Section III. The results and analysis are discussed in Section IV, and the paper is concluded in Section V. 


\section{Proposed Method}

The choice of PLC as a basis for the developed system is based on the following reasons [15], [33], [34]:

- Communication-based systems have a smaller NDZ and a higher fault detection speed compared with active and passive solutions.

- Economic feasibility of PLC compared with other wirecommunication-based methods. The means of communication is already present and available on the grid.

- Fault detection functionality can be implemented together with data transmission.

The proposed PLC system is evaluated using SDRs in the laboratory setup. Two USRP N210 and one USRP N200 SDR units, which are produced by Ettus Research company, were applied. An SDR is a radio system in which components commonly implemented in hardware (e.g. mixers, filters, amplifiers, (de)modulators) are implemented with software on hardware, thus providing high flexibility. A design of the PLC modem to be applied in the final solution, as well as final signaling settings and functionality of the system can be determined based on the system evaluation tests presented in the study. Essential constraints that have to be considered when designing a PLC-based solution are the grid topology and dimensions, the coupling interface, channel attenuation and noise in the channel, signaling frequency bandwidth, and signaling standard(s), including transmission power limitations.

\section{A. Signaling Scheme}

To detect islanding, continuous signaling is performed over the power line from MV grids to the customers and DGs on LV grids using PLC devices. These devices operate as transmitters $(\mathrm{Tx})$, receivers $(\mathrm{Rx})$, and intermediate transceivers $(\mathrm{T} x / \mathrm{Rx})$.

The main transmitter is installed at the primary substation in the MV grid, as illustrated in Fig. 1. This substation is also equipped with a signal receiver. Intermediate transceivers are installed at each MV/LV transformer, which is a component that degrades signal propagation by producing high signal attenuation and distortion [35]. A distribution transformer can be modeled by parasitic inductances and capacitances, and the characteristics of the transformer applied in this paper were previously studied in [36]. Therefore, a bypassing scheme (Fig. 2) is proposed [37]. The transceivers are powered from the LV side. Each customer on the LV grid is provided with a signal receiver, and each DG has both signal receiver and transmitter (Fig. 1). Each transmitter sends a modulated signal, which includes a personal identifier of the transmitter. This way receivers are able to detect what transmitters are connected to the signalling medium at the moment and, therefore, evaluate what grid components (DGs, transformers) are connected to the grid. This way intended islanding can be safely performed. Transmitter identification also improves the fault location accuracy.

The system flowchart is presented in Fig. 3. The primary substation is the main signal transmitter, and transmits the signal to MV grid and its branches. Each MV/LV transformer receives the signal from the primary substation and from all connected DGs. MV/LV substation processes the signals and transmits its own signal to customers and all connected DGs. DGs receive signals from the MV/LV transformer and other DGs installed in the LV grid. Then they transmit their corresponding signals. Customers operate only as signal receivers. Data from DGs and MV/LV transformers is then transmitted to the primary substation, allowing to perform continuous grid monitoring. Local grid monitoring is also performed at all DGs and $\mathrm{MV} / \mathrm{LV}$ transformers, as the described communication scheme allows them to evaluate the current grid state.

In general, multiuser signal transmission requires a certain method of channel division; for instance time-division multiplexing or frequency-division multiplexing can be applied. For DSSS modulation a code division approach is considered. Therefore the DSSS system transforms into a Code-Division Multiple Access (CDMA) modulation system. Such modification requires application of mutually-orthogonal spreading codes (used for signal modulation) for every signal transmitter. Receivers can switch between the codes and this way receive data from several transmitters. As demodulation is performed on a software level, several channels can be demodulated simultaneously, meaning that each signal receiver can receive and process data from all transmitters at the same time.

The signal reception algorithm, implemented both in the receiver and the transceiver, includes a signal processing mechanism, which evaluates the received message and signaling parameters (BER, bit rate, latency between packets). By analyzing the received signal parameters, or if no signal is heard, a loss of signal can be detected, which indicates that a channel between the current receiver and the transmitter is interrupted. If a warning message is received, it indicates that a grid fault has occurred before the previous transceiver. In this way, signal receivers can estimate the current grid state. In the

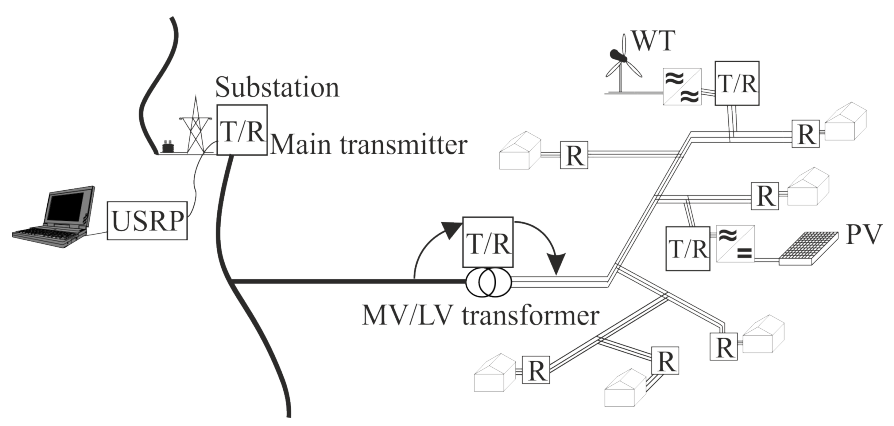

Fig. 1. MV/LV power grid with an integrated PLC-based LoM system [32].

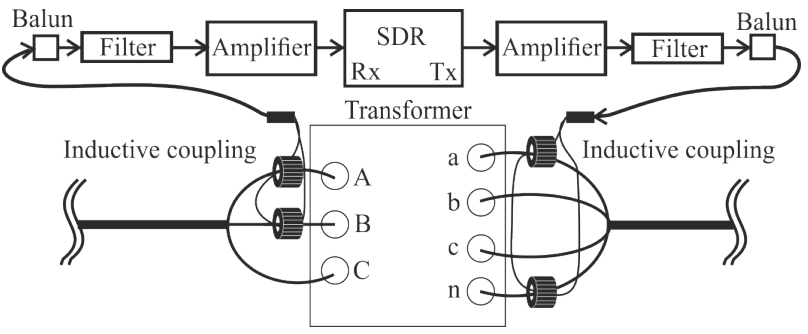

Fig. 2. Intermediate $\mathrm{Tx} / \mathrm{Rx}$ and bypassing setup installed at the transformer. 


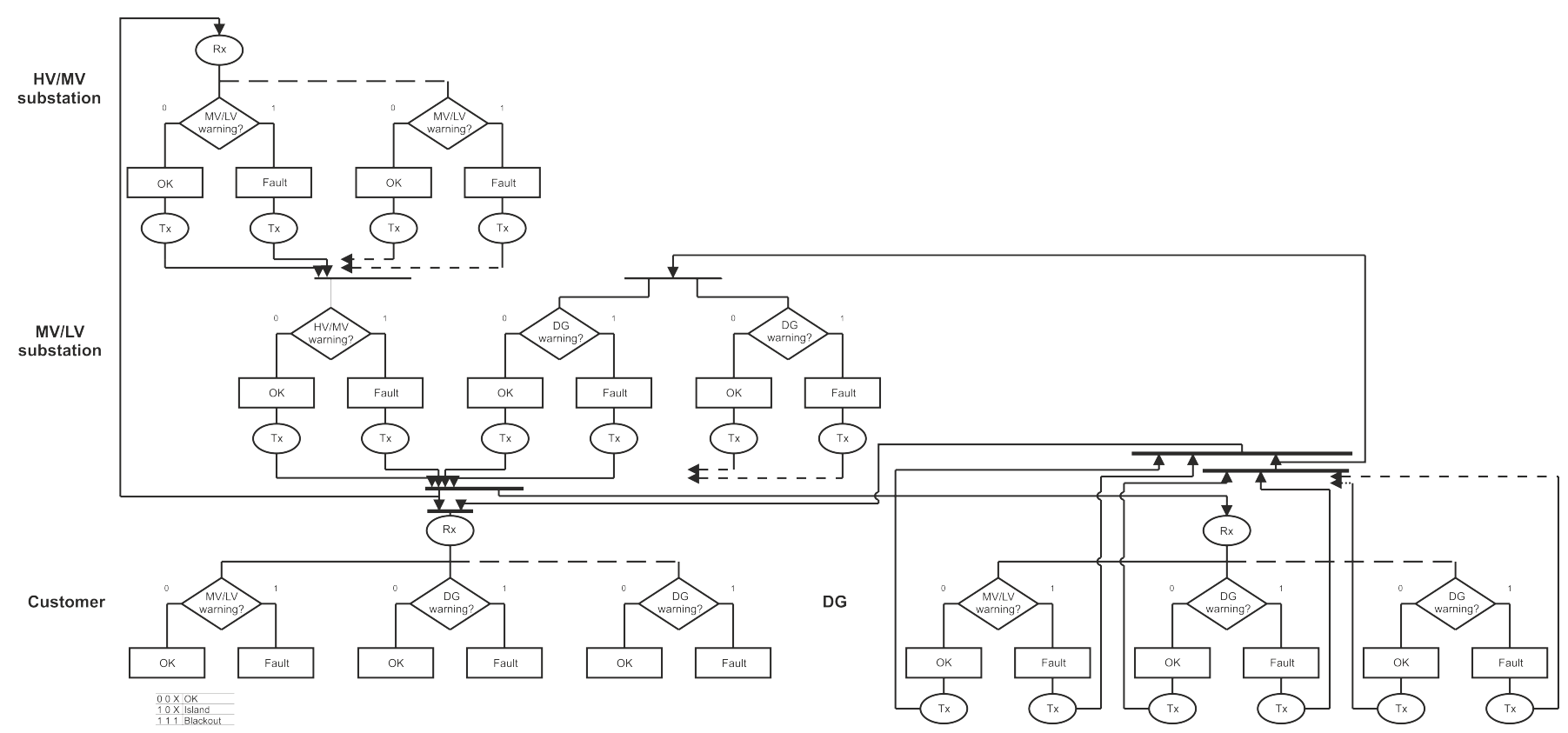

Fig. 3. Islanding detection system operational flowchart, including the main substation, MV/LV transformers, customer households and DG units.

case of islanding, it is then possible to disconnect a load/DG from the grid. In the case of an intended island scenario, it is possible to switch a DG to a controlled island mode and operate islanded grid segment as a $\mu \mathrm{G}$.

Application of the transformer bypass scheme has been debated in the literature, and a number of approaches have been presented. When bypassing is not considered, a transformer channel analysis is performed in order to choose a signaling frequency band with the lowest attenuation [35]. Moreover, conventional single carrier modulation techniques are often substituted by communication based on wideband modulation and channel access technologies, such as DSSS and orthogonal frequency division multiplexing (OFDM) [35], [37]. Bypassing approaches can use wireless communication or a bypassing communication channel with additional coupling components [35], [38]. In the proposed system, the bypassing setup comprises the PLC coupling interface placed on the MV side, which receives the signal (Fig. 2). Signal processing is performed in the PLC device and a new signal is injected through the interface installed to the LV side of the transformer. Of the two coupling interface alternatives, that is, capacitive and inductive, an inductive one is chosen because of the ease of installation and galvanic isolation [39].

\section{B. Frequency Band Selection}

The selection of a frequency band for the signaling system is based on consideration of the power line channel characteristics, noise in the channel, and relevant standards/legislation.

Signal attenuation in a power line is described by an attenuation coefficient $\alpha$, which increases as a function of frequency [40]. Moreover, $\alpha$ depends on the characteristics of conductors and insulation materials used in the power lines; overhead lines and underground cables. Typical insulation materials of underground LV power cables are for instance polyvinyl chloride (PVC) and cross-linked polyethylene (PEX). Different insulation materials produce different dielectric losses and thereby different signal attenuation [40], [41]. Considering the grid topology, MV line lengths can be several tens of kilometers while LV lines are typically only a few kilometers long. Further, the MV part of the grid is less branched, which is beneficial as the signaling power is divided into each branch and the channel capacity decreases [42].

Taking these aspects into account, the application of intermediate transceivers is vital in order to provide signaling through MV and LV grids. As $\alpha$ increases as a function of frequency, the low-frequency band $(<500 \mathrm{kHz})$ over the high frequency band is more suitable for LoM signaling [40].

Noise in the channel can be categorized into five noise types: colored background noise, narrow-band noise, periodic impulsive noise (synchronous and asynchronous to the mains frequency), and asynchronous aperiodic impulsive noise [40], [43]. Noise components are active in different frequency bands, but the frequency range below $20 \mathrm{MHz}$ can be chosen as the most suitable one for PLC considering noise scenarios [40]. Moreover, channel noise can be characterized as endogenous and exogenous, i.e. self-imposed and induced noise. For nonshielded MV lines exogenous noise is dominating [44].

Signal attenuation and the noise power spectral density (PSD) determine signal-to-noise ratio (SNR). A higher SNR with available bandwidth $B$ results in a higher channel capacity $C$, as seen in the following derived from Shannon's law

$$
C=B \cdot \log _{2}(1+S N R) .
$$

Based on the signal attenuation and noise scenarios under 
consideration, a frequency range below $500 \mathrm{kHz}$ is chosen as the signaling band for the system. PLC is regulated by CENELEC EN 50065-1 in the bandwidth of 3-148.5 kHz and by the FCC standard in the bandwidth of $14-480 \mathrm{kHz}$ [45], [46]. Moreover, a G3-PLC Physical Layer Specification is developed for modems, which operate in the frequency band of $35.9-90.6 \mathrm{kHz}$. This specification has provisions to extend the band to $480 \mathrm{kHz}$ [47]. As a result, and following the previous study [32], the frequency band of $100-300 \mathrm{kHz}$ is selected, and accordingly, the system performance is tested and evaluated with this signaling band.

\section{DSSS Modulation Technique}

In the proposed system, continuous signaling is implemented by applying DSSS on PLC devices. DSSS is a spread spectrum modulation technique, which withstands signal attenuation and noise in the channel more effectively than conventional single carrier modulation techniques [48]. DSSS is able to detect the signal with a negative SNR in the receiver [48].

DSSS modulation suggests phase shifting of a sine wave using code symbols - pseudonoise bit sequences, also called chips. Demodulation is possible only if these codes are known to the receiver. In this paper these code symbols, applied to spread the initial signal, are called spreading codes. A DSSS-based communication system (Fig. 4) is developed in a LabVIEW environment [32]. The original system consists of DSSS Tx and Rx schemes and is advanced by a Tx/Rx signal repeater application for the current work. The Tx/Rx demodulates the received signal, processes its parameters, modulates a new signal, and transmits it. The DSSS implementation in the study is based on binary phase-shift keying (BPSK). The main DSSS signaling parameters, such as spreading code length (SC) and synchronization key length (SK) can be adjusted by software. A synchronization key is a bit sequence placed in the beginning of each data packet and used for synchronization. The transmitted data packet has a length of 14 random bits, and the SK can be 8 and 12 bits. The SC has six options: Barker codes of 5, 7, 11, and 13 bits, and Walsh-Hadamard codes of 16 and 32 bits. Barker codes are often deployed in DSSS applications, as they provide low auto-correlation and low cross-correlation with other bit sequences [49]. Moreover, a 11 bit Barker code is applied in the 802.11b standard. Walsh-Hadamard codes, being mutually orthogonal, are often deployed in CDMA applications, which is a multiuser form of the DSSS [48]. The developed transmitter, transceiver repeater, and receiver applications, called virtual instruments (VIs) in LabVIEW, are each executed on a separate laptop, which is connected to a corresponding SDR.

\section{System Functionality}

1) Fault detection is based on signal processing. Signal parameters fluctuate within a range of certain limit values under normal conditions, and exceeding these limits is interpreted as a fault. The limits are determined by a healthy channel estimation, and are dependent on the channel conditions.

2) Fault location is interpreted by the received signal in the transceivers. An intermediate $\mathrm{Tx} / \mathrm{Rx}$ may detect a faulty segment in the grid, and send an error message or information about the grid condition downstream to the LV grid. As transceivers are installed at every $\mathrm{MV} / \mathrm{LV}$ transformer, each MV branch can be monitored.

\section{Fault Detection, Sensitivity Analysis, And Transformer Bypass Performance Assessment}

\section{A. Fault Detection Tests}

To evaluate signal processing as a base for fault detection in the proposed system, a series of tests were performed. The second target was to estimate the fault detection time and its compliance with the requirements set in [8], [9]. The laboratory setup for the tests is illustrated in Figs. 5 and 6; the receiver was coupled on the MV side of the MV/LV transformer. In this way, the PLC channel was deployed without signaling through the MV/LV transformer, but between the two transformers. The test was performed over the AXMK power cable with a $2 \mathrm{~dB}$ attenuator. During the test SNR was $12 \mathrm{~dB}$. For the transmitter, a signal amplifier TS200 by Accel Instruments was used. Bandpass filters (pass band of $140-500 \mathrm{kHz}$ ) were applied; the schematic of the filters is presented in Fig. 7. A receiver amplifier was also used at the receiver SDR (Fig. 8). The signaling center frequency was $200 \mathrm{kHz}$ and the bandwidth was $200 \mathrm{kHz}$. The DSSS communication system presented in [32] was used with an SC of five bits and an SK of eight bits.

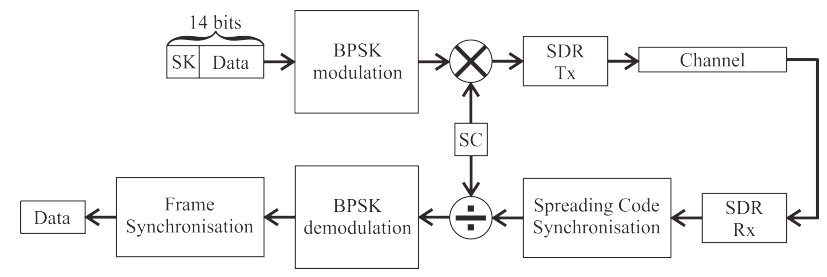

Fig. 4. Block diagram of the implemented DSSS algorithm into SDRs [32].

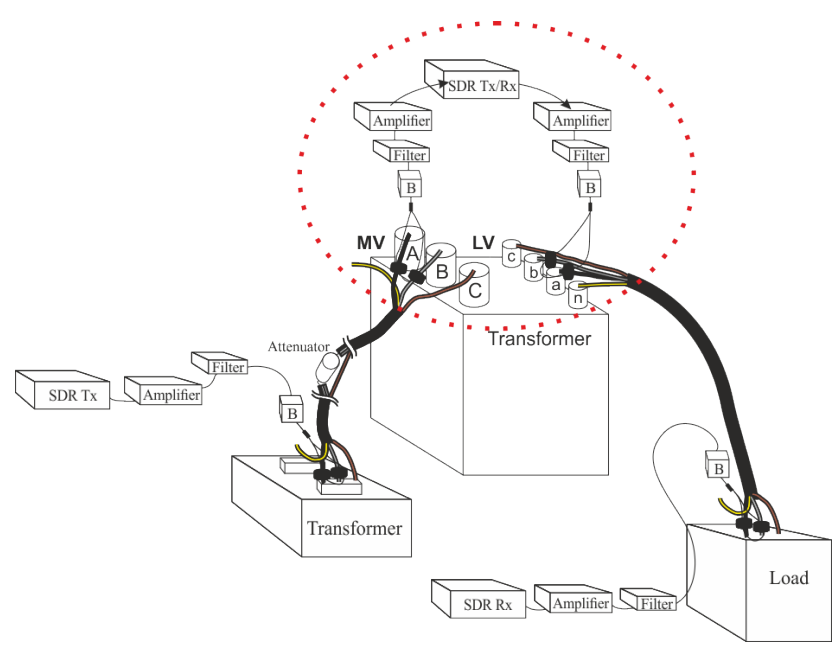

Fig. 5. Laboratory test setup used for the fault detection, DSSS sensitivity analysis, and bypassing signaling tests. Signaling is performed in the MV side of the transformer. An optional bypassing circuit, including coupling interfaces and radio equipment, is presented in the dashed circle. 
Signaling was carried out cyclically in an automated manner; first, the signal transmission was performed for $4 \mathrm{~s}$, and then, the transmitter was in the idle mode for $1 \mathrm{~s}$. The idle mode represented a LoM fault scenario, when signaling was interrupted. The signal receiver was continuously performing signal reception, measuring the received signal parameters and exporting them (at $15 \mathrm{~s}$ intervals). The receiver provided BER, bit rate, and processing latency values with a $1 \mathrm{~ms}$ resolution for the whole test period. In order to investigate if the fault point can be distinguished, time periods of $1 \mathrm{~s}$ before, $1 \mathrm{~s}$ of the fault period (idle mode), and $1 \mathrm{~s}$ after the fault were analyzed. The mean value of each parameter during the respective period in each test cycle was calculated and presented in Fig. 9.

As can be seen, the latency characteristic provided the highest sensitivity to a fault among the parameters under consideration. In the case of a loss of the signal, the mean processing latency rapidly increased from $23.8 \mathrm{~ms}$ to $89.3 \mathrm{~ms}$, and after the fault, it decreased to a value of $27.7 \mathrm{~ms}$ (Fig. 9). During the loss of the signal, the channel noise is interpreted as a signal. This issue intensifies with short SCs, as a smaller number of consecutive noise bits can be interpreted as a DSSS packet. The BER increased in the case of a fault, and the bit rate decreased. This can be explained by the fact that they were measured in $15 \mathrm{~s}$ time periods, not considering each

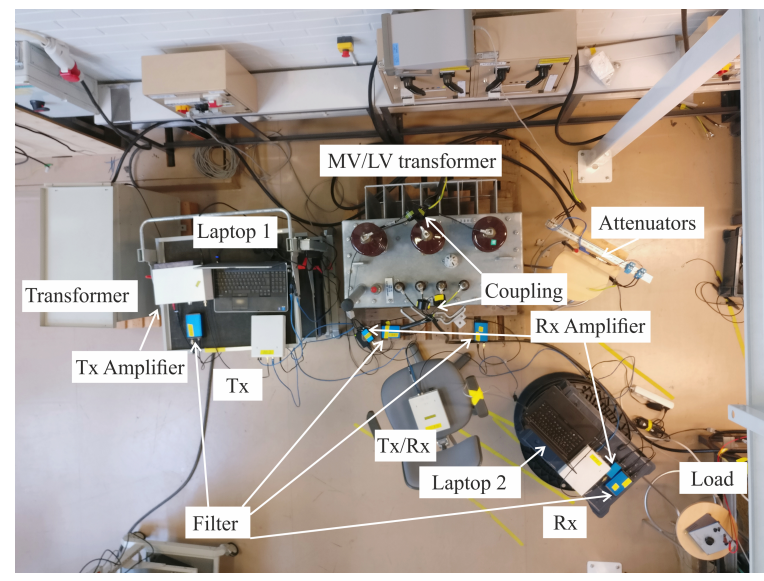

Fig. 6. Test setup build in the laboratory. Tx - a transmitter SDR, Rx - a receiver SDR, Tx/Rx - a transceiver SDR.

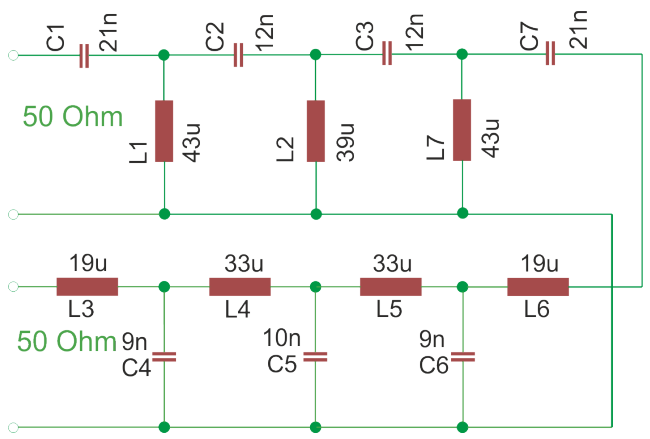

Fig. 7. Schematic of the PLC band-pass filter. The pass band is between 140 $\mathrm{kHz}$ and $500 \mathrm{kHz}$. received data packet separately. This approach is due to the calculation power limitation of the controlling PC. Both BER and bit rate parameters fluctuated less than the latency. Bit rate parameter has a noticeably lower change rate between the fault and after-the-fault states comparing to BER and latency parameters. Therefore it can be considered the least feasible for fault detection. It can be concluded that the following algorithm should be applied (Fig. 10): first, latency, as the most sensitive parameter is evaluated, then if the warning limit is exceeded, the BER parameter is evaluated in the derivative form and compared to the corresponding limit value. If the second limit is also exceeded, it means that the grid is in the island mode.

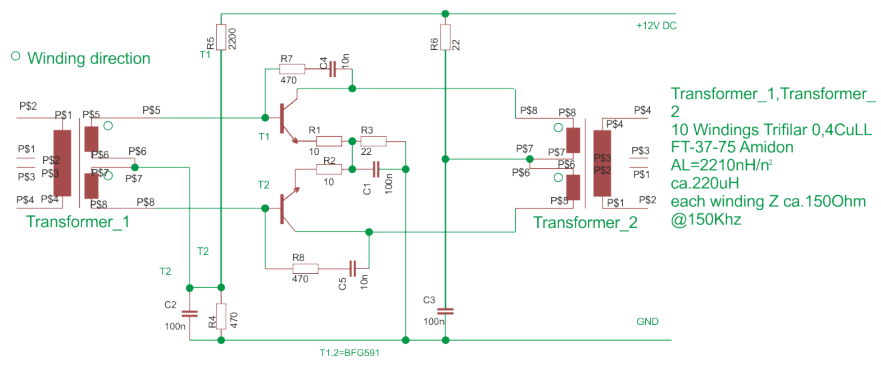

Fig. 8. Schematic of the PLC signal amplifier at the receiver.
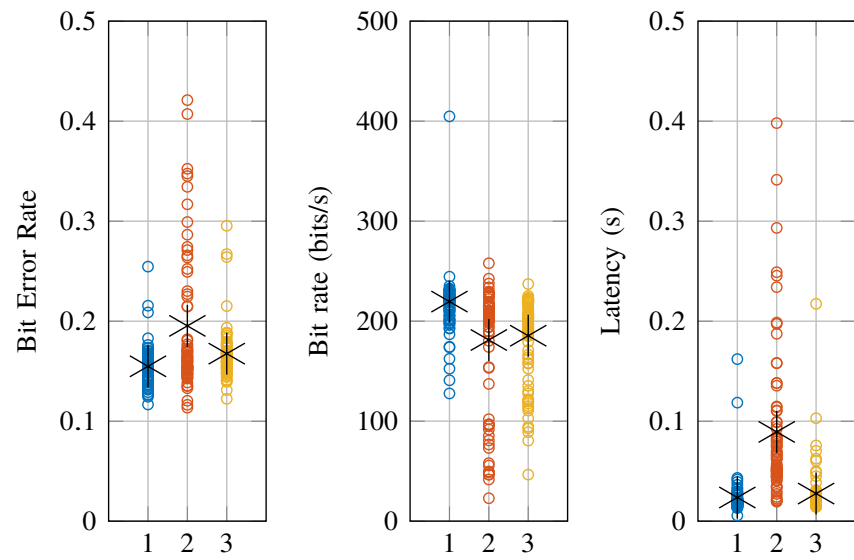

Fig. 9. Bit error rate, bit rate, and latency measurements during three periods, marked by numbers on the plots: $1-1 \mathrm{~s}$ before a fault; 2 -during a $1 \mathrm{~s}$ fault; 3 $-1 \mathrm{~s}$ after the fault. The asterisks indicate the mean values during the period.

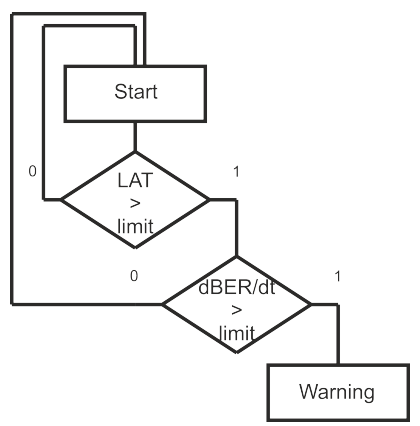

Fig. 10. Islanding detection algorithm, performed by the signal receiver. 
As the mean processing latency under normal operation conditions is $23.8 \mathrm{~ms}$, exceeding this limit can be considered a fault. Nevertheless, it should be noted that a faulty packet may be received under normal operation conditions as a result of a fluctuation in channel conditions (e.g. impulsive noise; load impedance variation causing impedance mismatch). Therefore, a certain number of consecutive faulty packets, which is interpreted as an islanding condition, should be set in the fault detection algorithm. Depending on this number, the fault detection time may differ, being a multiple of $23.8 \mathrm{~ms}$ (latency for a single packet) in the conditions under study. The obtained ID speed is comparable with the PLC-based solutions available and meets the requirements of the applicable standards [8], [9], [15], [29], [30]. Moreover, as observed in [32], the signal processing latency and thereby the fault detection speed and sensitivity, when deploying a channel with constant characteristics, mainly depend on the settings of the DSSS signaling. Different SC and SK can provide a clearer distinction between normal and fault conditions when the signaling parameters are monitored, also minimizing the issue of the channel noise being interpreted as a signal. Moreover, different DSSS parameters can provide a different system applicability range considering SNR.

\section{B. Sensitivity Analysis}

A DSSS sensitivity analysis is carried out to investigate system's performance against variation of a signal-to-noise ratio (SNR) - changing of BER,bit rate and latency parameter values, while the SNR and the DSSS settings (SC and SK) and modified.

It is important to notice that the SNR varies both during normal grid operation as a result of inconstant channel conditions, and in the case of islanding when the signaling is interrupted. Sensitivity is estimated by measuring the received signal parameters; BER, bit rate, signal latency with different DSSS settings on different SNR levels. Consequently, this analysis allows to determine the DSSS settings, i.e., the SC and SK, which provide the highest fault detection speed and communication throughput under various SNRs.

The sensitivity analysis was performed in the test setup presented in Fig. 5, when bypassing was not used. Six alternative SCs and two alternative SKs were used (Fig. 11). A signal attenuator, added to the channel, was used to adjust the SNR. In the tests, attenuations of $2,5,8,10,12,14,16,20,25$, and $27 \mathrm{~dB}$ were added, providing an SNR range of $-13-12 \mathrm{~dB}$. After adding the attenuator to the setup, the tests were run automatically in accordance with the program (Fig. 11) with all possible DSSS settings for approximately 12 hours. Then, the test was paused, and the SNR was decreased by adding more attenuators to the channel. After that, the tests were continued in an automated manner for 12 hours at each SNR level.

\section{Sensitivity Analysis Test Procedure}

The measurement results for BER, bit rate, throughput, and latency with $\mathrm{SK}=8$ and $\mathrm{SK}=12$ are presented in Fig. 12. In each plot measurement results under different SCs are provided.
1) BER: As can be seen for both SK options, a longer $\mathrm{SC}$ provides a lower error rate over the whole SNR range. Moreover, the settings with a longer SK provide a lower BER than the measurements with the same SC. The shorter the $\mathrm{SC}$, the higher the probability that random noise bits can be interpreted as an actual spreading code. If the SK is short, then there is a high probability that noise bits are interpreted as valid synchronization key and following bits are considered as valid data. This way short SC and SK contribute to a higher BER.

2) Bit rate: We can see that the settings with $\mathrm{SK}=12$ provide lower bit rate values than the settings with $\mathrm{SK}=8$. For $\mathrm{SK}=8$ longer SCs provide a lower bit rate, while for $\mathrm{SK}=12$ longer SCs provide a higher bit rate. This could be explained by the receiver with a short SC interpreting noise in the channel as a transmitted signal, which increases both the BER and bit rate. Moreover, a lower SK increases the number of data bits in the packet, which increases the bit rate.

3) Throughput is used to combine the obtained BER and bit rate measurements and achieve a more complete signal characteristic. Throughput reflects the number of correct data bits received per second. The throughput values are calculated

$$
\text { Throughput }=(1-2 \cdot \text { BER }) \cdot \text { BitRate. }
$$

A longer SC contributes to a lower BER, and a shorter SK means a higher number of data bits in each transmitted packet and thereby a higher bit rate. Both statements are valid for the throughput characteristic.

4) Latency describes the time elapsed between two received and processed data packets, thus being crucial for determining the fault detection time of the proposed system. For $\mathrm{SK}=8$ the shorter SCs provide a lower latency. The difference between the characteristics decreases with an increase in SNR. For SK=12 the difference between the characteristics is less noticeable, and several intersections can be seen. Considering the mean latency values and the small difference between the results, it can be concluded that longer SCs provide a lower latency, which is opposite to the scenario for $\mathrm{SK}=8$. Comparing these two graphs, it can be seen that the latency values for $\mathrm{SK}=8$ are lower, which means that a shorter SK provides a lower packet processing latency.

\section{Bypass Technique Assessment}

A test setup presented in Fig. 5 was used for the bypass scheme evaluation tests. Unlike in the scheme presented in Fig. 2, no transmitter amplifier was used in the bypassing.

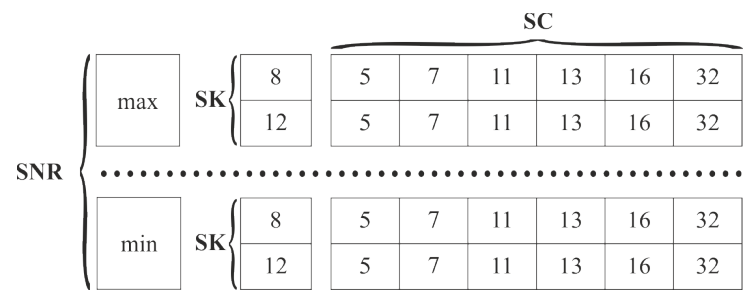

Fig. 11. Lab test procedure. Six SCs and two SKs are deployed for signaling on each SNR level. 

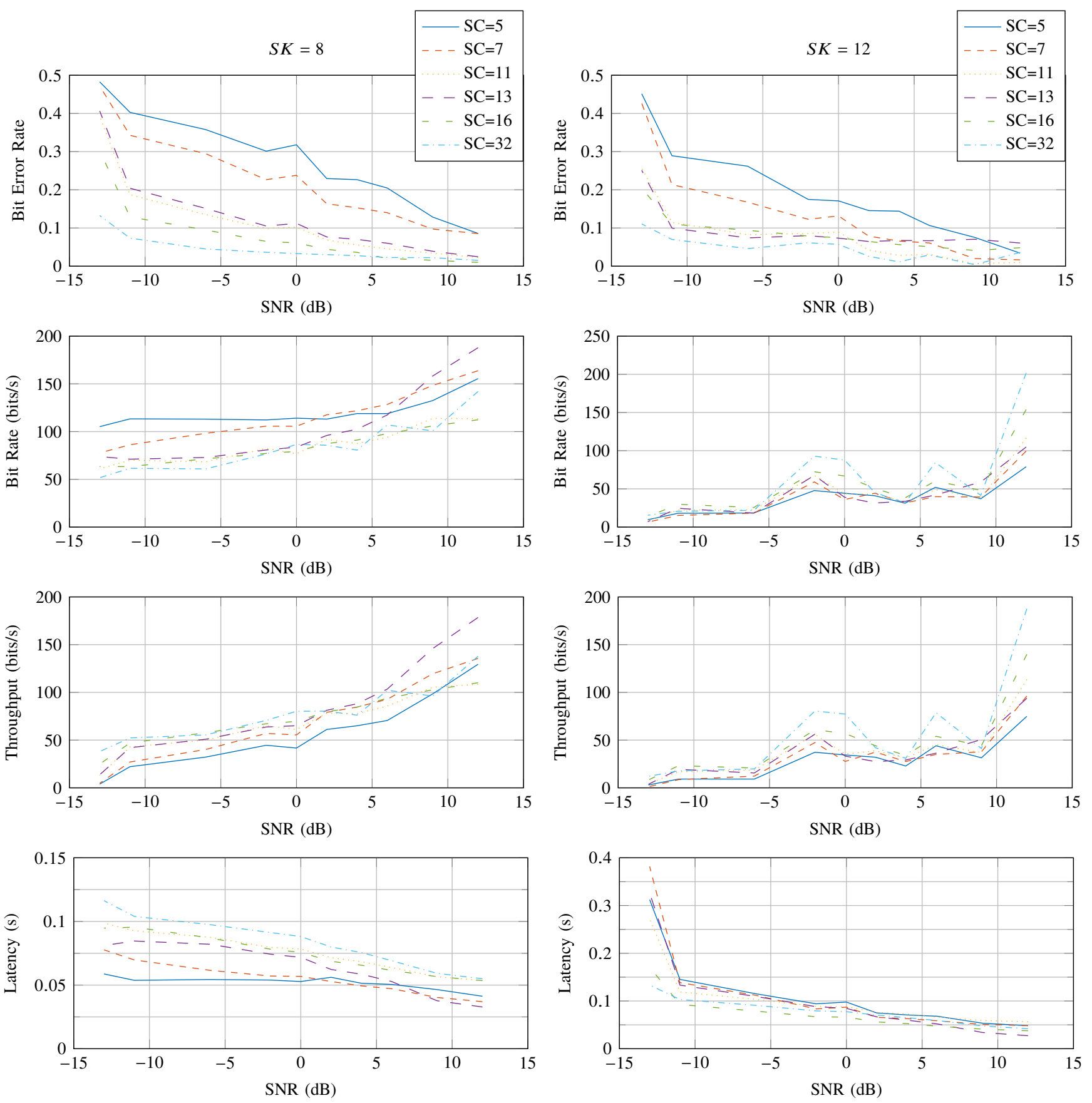

Fig. 12. Measurement results for different settings of SC and SK under different SNR values. Left column; SC=8 bits, right column; SC=12 bits.

Based on the sensitivity analysis, a setting of $(\mathrm{SC}=13, \mathrm{SK}=8)$ was chosen and applied in each SDR. The aim of the tests was to demonstrate in which channel conditions (considering SNR) bypassing can enhance fault detection, in other words, increase the detection speed and provide a higher throughput. Moreover, the target was to determine the conditions in which it is vital to apply the bypassing. The latency in the intermediate SDR was a source of concern, as the additional processing latency may decrease the total fault detection speed.

As in the sensitivity analysis, the signal parameters were monitored during several measurements with different signal attenuation values, and thus, under different SNR conditions. To compare the operation with and without a bypassing, the obtained characteristics were presented in the same figures with the original ones, whereas the SNR values were presented for the original channel (without bypassing). The throughput and 
latency characteristics are presented in Fig. 13.

The throughput characteristic was calculated with (2). The point with the highest SNR (12 dB in the initial channel) excluded, the bypassing scheme provides a higher throughput. The latency characteristic sums the processing latency in the transceiver (reception+transmission) and the receiver SDR. Similar to the throughput characteristic, the latency characteristic is less fluctuating than the characteristic for the conventional signaling scheme, and the signal latency is lower when bypassing is used. Nevertheless, with the SNR levels higher than $4 \mathrm{~dB}$ (in the original channel), the processing latency for bypassing was higher.

\section{Results and Analysis}

\section{A. Parameter Estimation}

The DSSS setting to be used in the system has to provide the highest fault detection speed and throughput among all tested settings. Fault detection speed is defined by the processing latency. Therefore, throughput and latency characteristics obtained during the sensitivity analysis were considered.

When considering the throughput, the setting $(\mathrm{SC}=13$, $\mathrm{SK}=8$ ) provides the highest total average throughput, whereas the settings $(\mathrm{SC}=32, \mathrm{SK}=8)$ and $(\mathrm{SC}=16, \mathrm{SK}=8)$ provide slightly worse results. Taking into account the processing latency, the setting $(\mathrm{SC}=5, \mathrm{SK}=8)$ demonstrates the smallest average latency value, but also a little sensitivity to SNR, and thereby to LoM (Fig. 12). Thus, the setting $(\mathrm{SC}=5, \mathrm{SK}=8)$ can not be accepted for the system. Therefore, the setting ( $\mathrm{SC}=7$, $\mathrm{SK}=8$ ) becomes the most feasible one for the application, ahead of settings $(\mathrm{SC}=13, \mathrm{SK}=8)$ and $(\mathrm{SC}=16, \mathrm{SK}=8)$.

Finally, taking all the studied characteristics into account, we may conclude that the setting $(\mathrm{SC}=13, \mathrm{SK}=8)$ is the most beneficial one for the system in the test setup under study. Alternative and less preferable options are the settings ( $\mathrm{SC}=32$, $\mathrm{SK}=8)$ and $(\mathrm{SC}=16, \mathrm{SK}=8)$.

\section{B. Bypass Scheme Efficiency}

According to the obtained results, if the SNR level on the MV side of the transformer is below $4 \mathrm{~dB}$, a bypassing scheme has to be applied. The throughput may increase significantly, whereas the processing latency will decrease even if the latency in the transceiver SDR is taken into account. At the same time, with a higher SNR, we can see that the mutual interference of the original signal and the bypass signal results in a lower throughput and a higher latency.

The average processing latency reaches $33 \mathrm{~ms}$ with SNRs above $4 \mathrm{~dB}$, when the bypassing scheme is not used. With lower SNRs when the bypassing scheme is applied, the average latency is up to $58 \mathrm{~ms}$. The fault detection time is a multiple of the processing latency, and thus, the requirements set in [8], [9] are met in both cases. A throughput of 176 bits/s was achieved in the SNR range under study.

\section{Conclusion}

A novel NB-PLC-based anti-islanding system employing DSSS modulation for continuous signaling was proposed. The
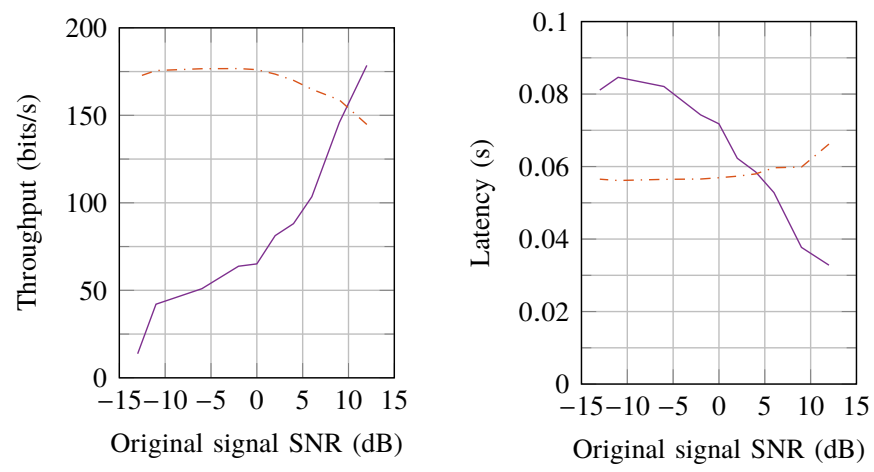

Without bypassing; -.-. With bypassing

Fig. 13. Comparing the measurement results for the signal throughput under different $\mathrm{SNR}$ values for $\mathrm{SC}=13 \mathrm{SK}=8$ in the setup with and without a bypass circuit with an intermediate transmitter.

developed fault detection algorithm based on the received signal analysis was validated by laboratory tests using SDRs. The achieved fault detection speed (min. $33 \mathrm{~ms}$ in the studied SNR range) meets the requirements of IEEE Std. 929-2000 and 1547-2003. Estimated signaling distance exceeds $10 \mathrm{~km}$. A DSSS sensitivity analysis was performed and settings $(\mathrm{SC}=13$ $\mathrm{SK}=8, \mathrm{SC}=16 \mathrm{SK}=8, \mathrm{SC}=32 \mathrm{SK}=8$ ) providing the highest fault detection speed and throughput were defined. The transformer bypassing scheme was tested and its feasibility for the signaling concept was proved. During the next phase of the concept development a multiuser communication applying the chosen DSSS settings in the CDMA configuration will be studied. System operation will be examined during consequential laboratory and field tests in the MV/LV grid.

Considering the study limitations, we may refer to the computation power of the PC in which the fault detection algorithm was executed. In order to minimize the influence of this component, longer test periods are needed. Moreover, the test measurements did not include or consider channel noise components, but only channel attenuation. Therefore, results have to be verified in distribution grids in the field.

\section{REFERENCES}

[1] B. Matic-Cuka and M. Kezunovic, "Islanding detection for inverterbased distributed generation using support vector machine method," IEEE Trans. Smart Grid, vol. 5, no. 6, pp. 2676-2686, Nov. 2014.

[2] Y. Guo and C. Zhao, "Islanding-aware robust energy management for microgrids," IEEE Trans. Smart Grid, vol. 9, no. 2, pp. 1301-1309, Mar. 2018.

[3] H. Zhang, L. Chen, H. Wang, Y. Zhao, L. Chen, and B. Duan, "Island partition of distribution network with microgrid and distributed generation based on floyd-warshall algorithm," in 2018 International Conference on Smart Grid and Electrical Automation (ICSGEA), June 2018, pp. 29-33.

[4] I. Kim and R. G. Harley, "A study on the intentional island formed by the residential photovoltaic system and the challenges to island operation," in 2015 North American Power Symposium (NAPS), Oct 2015, pp. $1-5$. 
[5] H. Laaksonen and P. Hovila, "Islanding detection during intended island operation of nested microgrid," in 2018 IEEE PES Innovative Smart Grid Technologies Conference Europe (ISGT-Europe), Oct 2018, pp. $1-6$.

[6] A. Esmaeilian and M. Kezunovic, "Prevention of power grid blackouts using intentional islanding scheme," IEEE Transactions on Industry Applications, vol. 53, no. 1, pp. 622-629, Jan 2017.

[7] D. Reigosa, F. Briz, C. B. Charro, P. Garcia, and J. M. Guerrero, "Active islanding detection using high-frequency signal injection," IEEE Trans. Ind. Appl., vol. 48, no. 5, pp. 1588-1597, Sep. 2012.

[8] IEEE Recommended Practice for Utility Interface of Photovoltaic (PV) Systems, IEEE Std 929-2000, Apr. 2000.

[9] IEEE Standard for Interconnecting Distributed Resources with Electric Power Systems, IEEE Std 1547-2003, Jul. 2003.

[10] H. Laaksonen, "Advanced islanding detection functionality for future electricity distribution networks," IEEE Transactions on Power Delivery, vol. 28, no. 4, pp. 2056-2064, Oct 2013.

[11] S. Shrivastava, S. Jain, R. K. Nema, and V. Chaurasia, "Two level islanding detection method for distributed generators in distribution networks," Electr. Power Energy Syst., vol. 87, pp. 222 - 231, 2017.

[12] A. Pouryekta, V. K. Ramachandaramurthy, N. Mithulananthan, and A. Arulampalam, "Islanding detection and enhancement of microgrid performance," IEEE Systems Journal, vol. 12, no. 4, pp. 3131-3141, Dec 2018.

[13] S. Murugesan, V. Murali, and S. A. Daniel, "Hybrid analyzing technique for active islanding detection based ond-axis current injection," IEEE Systems Journal, vol. 12, no. 4, pp. 3608-3617, Dec 2018.

[14] R. Anne, F. Katha Basha, R. Palaniappan, K. L. Oliver, and M. J. Thompson, "Reliable generator islanding detection for industrial power consumers with on-site generation," IEEE Transactions on Industry Applications, vol. 52, no. 1, pp. 668-676, Jan 2016.

[15] C. Li et al., "A review of islanding detection methods for microgrid," Renew. Sustain. Energy. Rev., vol. 35, pp. 211-220, Jul. 2014.

[16] A. G. Abd-Elkader, S. M. Saleh, and M. M. Eiteba, "A passive islanding detection strategy for multi-distributed generations," Electr. Power Energy Syst., vol. 99, pp. 146 - 155, 2018.

[17] M. M. Ostojić and M. B. Djurić, "The algorithm with synchronized voltage inputs for islanding detection of synchronous generators," Electr. Power Energy Syst., vol. 103, pp. 431 - 439, 2018.

[18] D. Bejmert and T. S. Sidhu, "Investigation into islanding detection with capacitor insertion-based method," IEEE Trans. Power Del., vol. 29, no. 6, pp. 2485-2492, Dec. 2014.

[19] Y. M. Makwana and B. R. Bhalja, "Experimental performance of an islanding detection scheme based on modal components," IEEE Transactions on Smart Grid, vol. 10, no. 1, pp. 1025-1035, Jan 2019.

[20] E. Serban, C. Pondiche, and M. Ordonez, "Islanding detection search sequence for distributed power generators under ac grid faults," IEEE Transactions on Power Electronics, vol. 30, no. 6, pp. 3106-3121, June 2015.

[21] Z. Ye, A. Kolwalkar, Y. Zhang, P. Du, and R. Walling, "Evaluation of anti-islanding schemes based on nondetection zone concept," IEEE Trans. Power Electron., vol. 19, no. 5, pp. 1171-1176, Sep. 2004.

[22] C. L. Wagner, W. E. Feero, W. B. Gish, and R. H. Jones, "Relay performance in dsg islands," IEEE Transactions on Power Delivery, vol. 4, no. 1, pp. 122-131, Jan 1989.

[23] S. D. Kermany, M. Joorabian, S. Deilami, and M. A. S. Masoum, "Hybrid islanding detection in microgrid with multiple connection points to smart grids using fuzzy-neural network," IEEE Transactions on Power Systems, vol. 32, no. 4, pp. 2640-2651, July 2017.

[24] O. N. Faqhruldin, E. F. El-Saadany, and H. H. Zeineldin, "A universal islanding detection technique for distributed generation using pattern recognition," IEEE Transactions on Smart Grid, vol. 5, no. 4, pp. 19851992, July 2014.

[25] D. Voglitsis, F. Valsamas, N. Rigogiannis, and N. P. Papanikolaou, "On harmonic injection anti-islanding techniques under the operation of multiple der-inverters," IEEE Transactions on Energy Conversion, vol. 34, no. 1, pp. 455-467, March 2019.

[26] Q. Sun, J. M. Guerrero, T. Jing, J. C. Vasquez, and R. Yang, "An islanding detection method by using frequency positive feedback based on FLL for single-phase microgrid," IEEE Trans. Smart Grid, vol. 8, no. 4, pp. 1821-1830, Jul. 2017.

[27] M. Ropp, K. Aaker, J. Haigh, and N. Sabbah, "Using power line carrier communications to prevent islanding [of pv power systems]," in 2000 IEEE Photovoltaic Specialists Conference (PVSC), 2000, pp. 16751678.

[28] M. Ropp, D. Larson, S. Meendering, D. McMahon, J. Ginn, J. Stevens, W. Bower, S. Gonzalez, K. Fennell, and L. Brusseau, "Discussion of a power line carrier communications-based anti-islanding scheme using a commercial automatic meter reading system," in 2006 IEEE 4th World Conf. on Photovoltaic Energy Conversion (WCPEC), vol. 2, May 2006, pp. 2351-2354.

[29] S. Voima, P. Valisuo, and K. Kauhaniemi, "Power line carrier based loss of mains protection in medium voltage distribution networks," in 2016 IET Int. Conf. on Development in Power System Protection (DPSP), Mar. 2016, pp. 1-6.

[30] G. Artale, A. Cataliotti, V. Cosentino, D. D. Cara, S. Guaiana, S. Nuccio, N. Panzavecchia, and G. Tinè, "Smart interface devices for distributed generation in smart grids: The case of islanding," IEEE Sensors Journal, vol. 17, no. 23, pp. 7803-7811, Dec 2017.

[31] A. Pinomaa, "Power-line-communication-based data transmission concept for an lvde electricity distribution network - analysis and implementation," in PhD Thesis, Lappeenranta university of Technology, Electrical Engineering, 2013.

[32] A. Poluektov, A. Romanenko, A. Pinomaa, J. Ahola, and A. Kosonen, "Sensitivity analysis of a PLC-based anti-islanding solution using DSSS," in 2017 IEEE Int. Symp. on Power Line Communications and its Applications (ISPLC), Apr. 2017, pp. 1-6.

[33] S. Galli, A. Scaglione, and Z. Wang, "For the grid and through the grid: The role of power line communications in the smart grid," Proc. IEEE, vol. 99, no. 6, pp. 998-1027, Jun. 2011.

[34] S. Canale, A. D. Giorgio, A. Lanna, A. Mercurio, M. Panfili, and A. Pietrabissa, "Optimal planning and routing in medium voltage powerline communications networks," IEEE Trans. Smart Grid, vol. 4, no. 2, pp. 711-719, Jun. 2013.

[35] W. C. Black, "Data transmission through distribution transformers without bypass components," in 2010 IEEE Int. Symp. on Power Line Communications and its Applications (ISPLC), Mar. 2010, pp. 13-17.

[36] A. Pinomaa, A. Poluektov, J. Ahola, and A. Kosonen, "Analysis of channel characteristics and modeling of a transformer for plc-based lossof-mains concept," in 2016 International Symposium on Power Line Communications and its Applications (ISPLC), March 2016, pp. 230235.

[37] A. Poluektov, A. Pinomaa, J. Ahola, and A. Kosonen, "Designing a power-line-communication-based lom protection concept with application of software-defined radios," in 2016 Int. Symp. on Power Line Communications and its Applications (ISPLC), Mar. 2016, pp. 156161.

[38] C. J. Kikkert, "MV to LV transformer plc bypass coupling networks for a low cost smart grid rollout," in 2011 IEEE PES Innovative Smart Grid Technologies, Nov. 2011, pp. 1-6.

[39] A. Kosonen and J. Ahola, "Comparison of signal coupling methods for power line communication between a motor and an inverter," IET Electric Power Applications, vol. 4, no. 6, pp. 431-440, Jul. 2010.

[40] M. Gotz, M. Rapp, and K. Dostert, "Power line channel characteristics and their effect on communication system design," IEEE Commun. Mag., vol. 42, no. 4, pp. 78-86, Apr. 2004.

[41] A. Pinomaa, J. Ahola, and A. Kosonen, "PLC concept for LVDC distribution systems," IEEE Commun. Mag., vol. 49, no. 12, pp. 55-63, Dec. 2011.

[42] J. Anatory, N. Theethayi, R. Thottappillil, M. M. Kissaka, and N. H. 
Mvungi, "Broadband power-line communications: The channel capacity analysis," IEEE Trans. Power Del., vol. 23, no. 1, pp. 164-170, Jan. 2008.

[43] M. Zimmermann and K. Dostert, "Analysis and modeling of impulsive noise in broad-band powerline communications," IEEE Trans. Electromagn. Compat., vol. 44, no. 1, pp. 249-258, Feb. 2002.

[44] A. N. Milioudis, K. N. Syranidis, G. T. Andreou, and D. P. Labridis, "Modeling of medium-voltage power-line communication systems noise levels," IEEE Trans. Power Del., vol. 28, no. 4, pp. 2004-2013, Oct. 2013.

[45] EN-50065-1, "Signalling on low-voltage electrical installations in the frequency range $3 \mathrm{kHz}$ to $148.5 \mathrm{kHz}$ part 1: general requirements, frequency bands and electromagnetic disturbances," CENELEC, 2001.

[46] FCC, "Title 47 cfr part 15 subpart b," Federal Communications Commission Std., 1998.

[47] “G3-PLC physical layer specification," Electricite Reseau Distribution, France, Aug. 2009.

[48] J. Meng and A. E. Marble, "Effective communication strategies for noise-limited power-line channels," IEEE Trans. Power Del., vol. 22 , no. 2, pp. 887-892, Apr. 2007.

[49] T. O'Farrell, "Design and evaluation of a high data rate optical wireless system for the diffuse indoor channel using barker spreading codes and rake reception [optical wireless communications]," IET Communications, vol. 2, no. 1, pp. 35-44, Jan. 2008. 